\title{
Effects of the Sonas Program on Anxiety and Depression in Nursing Home Residents with Dementia: A 6-Month Randomized Controlled Trial
}

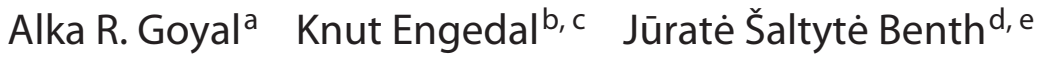 \\ Benedicte Sørensen Strøm ${ }^{f}$ \\ ${ }^{a}$ Faculty of Health Sciences, Department of Nursing and Health Promotion, Oslo Metropolitan University, Oslo,

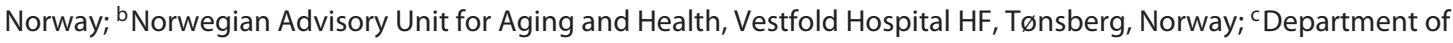 \\ Geriatrics, Oslo University Hospital, Oslo, Norway; ${ }^{\mathrm{d} I n s t i t u t e}$ of Clinical Medicine, University of Oslo, Oslo, Norway; \\ ${ }^{\mathrm{e}}$ Health Services Research Unit, Akershus University Hospital, Lørenskog, Norway; ${ }^{\mathrm{f}}$ Faculty of Health Studies, VID \\ Specialized University, Oslo, Norway
}

\section{Keywords}

Dementia · The Sonas program · Anxiety · Depression · Nursing home

\begin{abstract}
Introduction: There is a dearth of evidence about the effects of Sonas, a multisensory stimulation on people with dementia (PWD). The main aim of this study was to examine the effects of the Sonas program on anxiety and depression in nursing home $(\mathrm{NH})$ residents with dementia. Methods: In all, 120 PWD $\geq 65$ years of age from $6 \mathrm{NHs}$ were included in a randomized control trial and were allocated to 1 of 3 groups for 24 weeks: a Sonas program group $(n=48)$, a reading group ( $n=32)$, and a control group $(n=40)$. One hundred and five participants completed follow-up assessments. Anxiety and depression were assessed by the Rating Anxiety in Dementia (RAID) scale and the Cornell Scale for Depression in Dementia (CSDD), respectively. Generalized linear mixed models were estimated to assess trends in the proportion of participants with anxiety (a RAID score $\geq 11$ ) and de-
\end{abstract}

karger@karger.com www.karger.com/dee

Karger $\stackrel{\text { '⿳亠丷厂 }}{ }$

BOPEN ACCESS
(C) 2021 The Author(s)

Published by S. Karger AG, Basel

This is an Open Access article licensed under the Creative Commons Attribution-NonCommercial-4.0 International License (CC BY-NC) (http://www.karger.com/Services/OpenAccessLicense), applicable to the online version of the article only. Usage and distribution for commercial purposes requires written permission. pression (a CSDD score $\geq 10$ ). Results: No significant reduction in anxiety from baseline to follow-up was observed in any of the groups. Participants in the Sonas group showed a significant reduction in depression from baseline to 12 weeks $(p=0.001)$ and from baseline to 24 weeks $(p=0.009)$. Conclusion: The Sonas program had no effect on severity of anxiety but a reduction in depressive symptoms was found in PWD.

(c) 2021 The Author(s) Published by S. Karger AG, Basel

\section{Introduction}

Anxiety and depression as symptoms at disease level are common conditions (comorbidities) in older people with dementia (PWD) and are more frequent in PWD living in nursing homes (NHs) than in those living at home [1-5]. Some studies report that anxiety and depression are more prevalent in early than in late stages of dementia $[6,7]$, but other studies report that anxiety and 
depression are equally prevalent in all stages of dementia but may be diagnosed as challenging behavior (especially as agitation) in the late stages [8-11]. Anxiety and depression can be difficult to diagnose in the late stages of dementia as patients may have difficulties in expressing their feelings verbally and also due to the overlapping symptoms of dementia, depression, and physical morbidities in older people [12-14]. Regardless, anxiety and/or depression can have many negative effects on PWD, their family, and professional caregivers, and thus needs to be diagnosed and treated [2, 15-17].

The interplay of biological and psychosocial factors could be the underlying causes of anxiety and depression as well as other neuropsychiatric symptoms (NPS) in PWD [18-20]. PWD undergo various changes in neuropsychological functioning, which adversely impact their ability to function independently, their communication, and their participation in social activities. This may lead to a lack of social engagement and to psychological problems like anxiety and depression [20,21].

Effective treatment in terms of medication for dementia due to Alzheimer's disease was first available in the early 1990s [22]. However, so far there is no cure for dementia, and, with the increasing prevalence of dementia worldwide, the World Health Organisation (WHO) recognizes dementia as a public health priority [23]. Studies show that the effects of pharmacological interventions to treat anxiety, depression, or other NPS of dementia are modest and, besides, the use of psychotropic medications can be associated with major side effects in PWD [24,25]. Therefore, psychosocial interventions should be the first step to treat anxiety, depression, and other NPS in PWD $[26,27]$.

Previous studies have investigated the effects of a variety of psychosocial interventions on anxiety and depression among PWD [27]. The Sonas program, a multisensory stimulation program developed by Sister Mary Threadgold in 1990, is primarily an intervention with the aim of promoting the ability of PWD to communicate [28]. Several studies have examined the effects of the Sonas program on communication, cognition, aggression, functions of activities of daily living, depression/mood, well-being, and quality of life [28]. However, the effects on anxiety have only been measured in 1 study; Hutson et al. [29] conducted a pilot study with a small number of participants $(N=39)$ and the intervention was short, with the Sonas program being applied for up to 7 weeks only. The study did not find any significant differences between the intervention and control groups in terms of anxiety. In all, 3 studies have been published that investi- gated the effects of the Sonas program on depression/ mood, and the findings were ambiguous [29-31]. The qualitative study by Dugmore [30] reported positive effects on the mood of participant as expressed by the staff. The study by Jackson et al. [31] found a significant increase in depressive symptoms in the intervention group after 8 weeks of the Sonas program and no significant changes in the control group. Hutson et al. [29] did not report any significant differences between the intervention and control groups in relation to depression.

As no definite knowledge exists as to whether the Sonas program does indeed have an effect on anxiety and depression in PWD, we wanted to investigate this in $\mathrm{NH}$ residents with moderate to severe dementia. We further wanted to explore whether the effects of this program on anxiety and depression could be related to the cognitive function of the residents.

\section{Materials and Methods}

\section{Participants and Study Design}

In all, 120 residents $\geq 65$ years of age and diagnosed with dementia from 6 NHs in Ireland were included in a randomized control trial (RCT). The participants were allocated to 1 of 3 groups for 24 weeks: (1) a Sonas program group $(n=48),(2)$ a reading group, regarded as an attention placebo group $(n=32)$, and (3) a control group with no intervention $(n=40)$. The flowchart of study participants is shown in Figure 1. The design with 3 groups was used to rule out whether the effects of the Sonas program could be caused by special attention given to the intervention group, rather by the intervention itself [32]. In addition, all participants received standard care and attended usual activities in the NH. Recruitment of participants, the randomization process, and data collection are described in detail elsewhere [33]. Data were collected at baseline before randomization (T0), after 12 weeks (T1), and after 24 weeks (T2).

\section{Brief Description of the Groups}

Sonas Group. The participating NHs had their own Sonas-licenced practitioner (SLP) who attended a 3-day training on how to conduct a Sonas session. Each NH had 1 Sonas group consisting of 8 participants. The groups were led by SLPs and an assistant. The intervention was carried out twice a week for 24 weeks, and each session lasted $45 \mathrm{~min}$. The Sonas program followed a fixed structure each time and consisted of 11 elements [33].

Multisensory stimulation in the Sonas program consists of the following elements:

- A signature tune and greeting song

- Exercise to music

- Smell

- A "sing-along" which includes 3 familiar songs

- Relaxation music/massage (the senses of taste and touch are stimulated)

- Music with a percussion instrument

- Joining-in proverbs 
Fig. 1. Flowchart of participants.

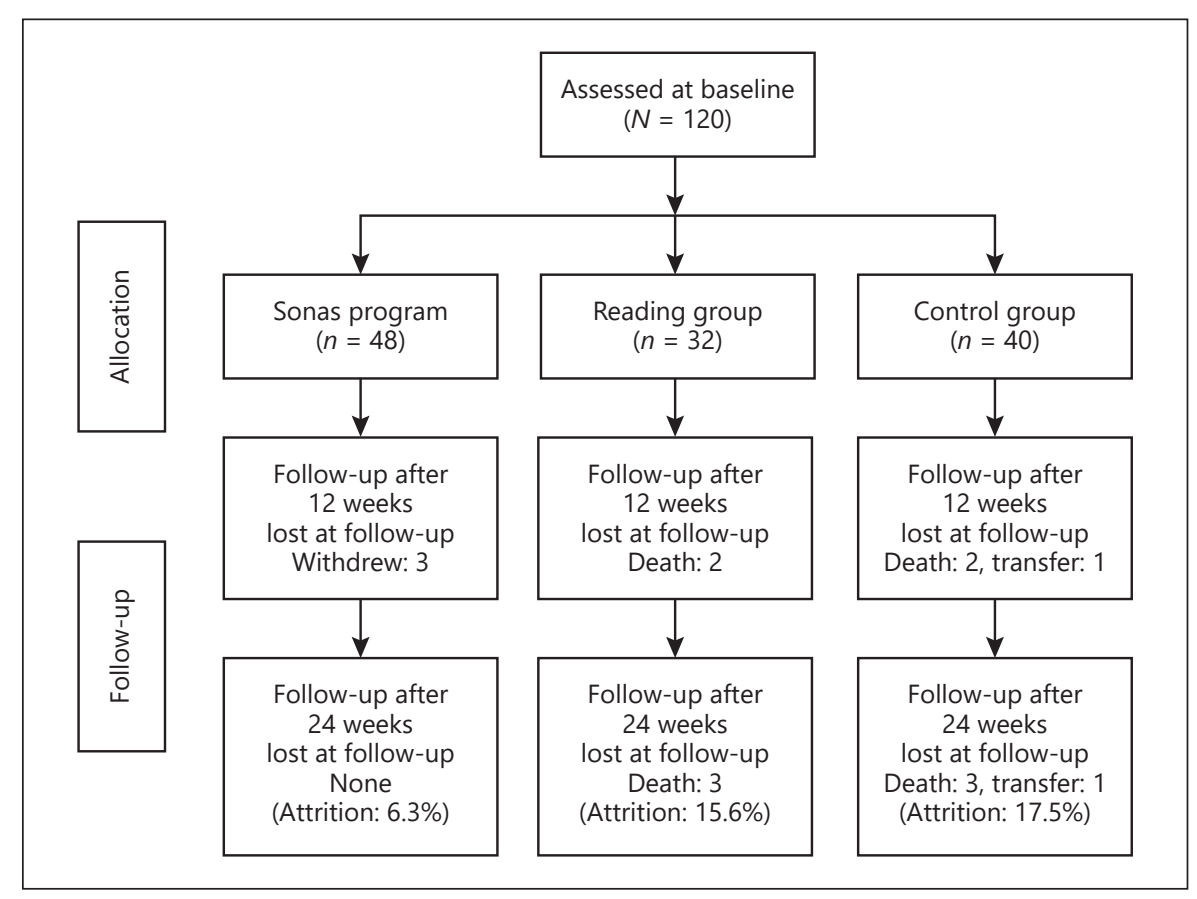

- Listening to poetry

- A contribution

- A Second "sing-along"

- The closing song and signature tune

Reading Group. The staff at the participating NHs were instructed in how to lead and conduct the intervention. The participants attended the reading group twice a week for 24 weeks, and each session lasted for $45 \mathrm{~min}$, while a staff member read from a newspaper.

Control Group. The control group continued with their routine activities provided by the $\mathrm{NH}$.

\section{Assessment Scales}

\section{Mini-Mental Status Examination}

Severity of participants' cognitive impairment was assessed with the Mini-Mental Status Examination (MMSE) screening test [34] which has a minimum score of 0 and a maximum score of 30. A higher score indicates higher cognitive function [34]. MMSE scores of 0-20 are classified as severe to moderate cognitive impairment. The MMSE has been judged to have satisfactory reliability as well as construct and criterion validity [35].

The Rating Anxiety in Dementia

The Rating Anxiety in Dementia (RAID) scale is a dementiaspecific anxiety scale and consists of 20 items, 18 of which can be scored from 0 (absent) to 3 (severe), or $U$ (unable to assess). The total score ranges from 0 to 54 , with higher scores indicating more severe anxiety. Two items (phobias and panic attacks) are assessed as present or absent and are not included in the total score [36]. The items are rated after interviewing the patient and care staff and checking the clinical notes from the previous 2 weeks. A score of $\geq 11$ suggests significant clinical anxiety [36].

Anxiety and Depression and the Sonas Program
The Cornell Scale for Depression in Dementia

The Cornell Scale for Depression in Dementia (CSDD) is an observation-based scale that is widely used to assess depressive symptoms in PWD [37]. The scale includes 19 items to for assessing a patient's level of depression based on caregivers' observations over the previous week. The rating scale is from 0 to 38 , where 38 is the highest score, i.e., denoting more severe depression [37]. A score of $\geq 10$ on the CSDD suggests significant clinical depression in PWD living in NHs [38].

\section{Data Analyses}

Demographic and clinical characteristics of participants were presented as means and standard deviations (SD) or $n(\%)$, as appropriate. Generalized linear mixed models were estimated to assess trends in the proportion of participants with anxiety (RAID score $\geq 11$ ) and depression (CSDD score $\geq 10$ ). The models contained fixed effects for time point (with baseline as reference), group (control as reference), and the interaction between time and group. A significant interaction would imply differences between groups in trends in the proportion of participants with anxiety or depression. Random intercepts for participants nested within NHs were included to account for within-patient and within- $\mathrm{NH}$ correlations. Random effects for NHs were skipped if no cluster effect was present as assessed by intraclass correlation coefficient. The results were presented graphically as odds ratios with $p$ values reported in the text. Due to the small group size, the proportions of participants with anxiety and depression stratified by level of cognition (MMSE scores of $0-10$ vs. 11-20) were presented descriptively and compared by $z$ test for proportions, whenever considered relevant. All tests were two-sided and $p<0.05$ was considered statistically significant. The statistical analyses were conducted using SPSS v26 and SAS v9.4. 
Table 1. Characteristics of participants at baseline

\begin{tabular}{lcccc}
\hline & $\begin{array}{l}\text { Total } \\
(N=120)\end{array}$ & $\begin{array}{l}\text { Sonas group } \\
(n=48)\end{array}$ & $\begin{array}{l}\text { Reading group } \\
(n=32)\end{array}$ & $\begin{array}{l}\text { Control group } \\
(n=40)\end{array}$ \\
\hline Women, $n(\%)$ & $93(77.5)$ & $40(83.3)$ & $25(78.1)$ & $28(70.0)$ \\
Men, $n(\%)$ & $27(22.5)$ & $8(16.7)$ & $7(21.9)$ & $12(30.0)$ \\
Age, years & $84.8(7.0)$ & $86.1(6.6)$ & $84.9(7.2)$ & $83.3(7.1)$ \\
MMSE & $9.0(7.1)$ & $7.7(6.7)$ & $10.0(7.2)$ & $9.7(7.5)$ \\
RAID sum score & $8.7(6.5)^{*}$ & $9.7(6.9)$ & $7.4(5.5) * *$ & $8.5(6.6)$ \\
CSDD sum score & $8.4(5.7)$ & $8.8(4.9)$ & $7.8(6.0)$ & $8.4(6.2)$ \\
Degree of cognitive function & & & & $21(52.5)$ \\
$\quad$ Moderate (MMSE 11-20) & $57(47.5)$ & $19(39.6)$ & $17(53.1)$ & $19(47.5)$ \\
\hline
\end{tabular}

Values express mean (SD), unless otherwise indicated. ${ }^{*} n=119$ ( 1 missing); ${ }^{* *} n=31$ ( 1 missing).

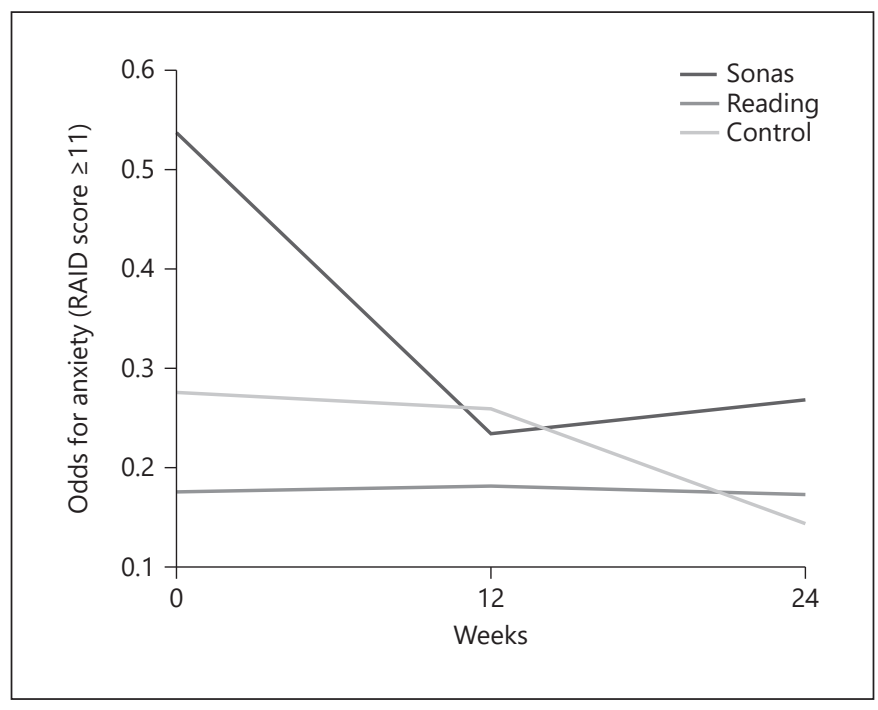

Fig. 2. The course of anxiety in the 3 groups (GLIMMIX model).

\section{Results}

There was a total of 120 residents with an average age of 85 years from 6 NHs, and 77.5\% were female. Participants had moderate to severe dementia with an average MMSE score of 9.0 (SD 7.1); 47.5\% had moderate dementia defined as an MMSE score of $11-20$ and $52.5 \%$ had severe dementia (an MMSE score $\leq 10$ ). Etiological dementia diagnoses were not known. Characteristics of the participants at baseline are shown in Table 1. The secondary analyses of the study by Strøm et al. [33] examine the effects of the Sonas program on anxiety and depression in $\mathrm{NH}$ residents with moderate to severe dementia.

\section{Effects on Anxiety}

Overall, there were no significant differences (nonsignificant interactions) between the groups regarding the trend in the proportion with anxiety. A nonsignificant reduction in anxiety from baseline (T0) to 12 weeks (T1) was observed in the Sonas group, but no changes were found in the reading and control groups (Fig. 2; Table 2).

When stratifying the groups according to MMSE score, no changes were found in the occurrence of anxiety in any group at any time point (Table 3 ).

\section{Effects on Depression}

There were no overall group differences (nonsignificant interactions) in the course of depression. Participants in the Sonas group showed a significant reduction in depression from baseline (T0) to 12 weeks (T1) $(p=$ $0.001)$ and 24 weeks (T2) ( $p=0.009)$. The increase in depression from $\mathrm{T} 1$ to $\mathrm{T} 2$ in the reading group was nonsignificant (Fig. 3). The proportions of participants with depression in the 3 groups from baseline to follow-up is shown in Table 4.

When stratifying the groups according to levels of cognition, no significant changes were found in the occurrence of depression in any group because there were too few cases to be able to calculate $p$ values at any time point (Table 5).

\section{Discussion}

The overall aim of this study was to examine whether the Sonas program had any effect on anxiety and depression in $\mathrm{NH}$ residents with moderate to severe dementia. 
Table 2. The mean RAID sum score (SD) and proportion of participants with anxiety (RAID score $\geq 11$ ) in the 3 groups at baseline and at follow-up

\begin{tabular}{llccc}
\hline Time point & Total & Sonas & Reading & Control \\
\hline T0 (at baseline) & & & & \\
$\quad N$ & $119^{*}$ & 48 & 31 & 40 \\
RAID score & $8.7(6.5)$ & $9.7(6.9)$ & $7.4(5.5)$ & $8.5(6.6)$ \\
$\quad$ Proportion, $n(\%)$ & $36(30.3)$ & $18(37.5)$ & $7(22.6)$ & $11(27.5)$ \\
T1 (at 12 weeks) & & & & 37 \\
$N$ & 113 & 46 & $6.8(4.9)$ & $8.0(5.3)$ \\
Raid score & $8.1(5.2)$ & $8.9(5.2)$ & $7(23.3)$ & $10(27.0)$ \\
Proportion, $n(\%)$ & $30(26.5)$ & $13(28.3)$ & & 33 \\
T2 (at 24 weeks) & & 45 & 27 & $7.5(4.5)$ \\
$N$ & 105 & $8.6(5.8)$ & $7.2(5.9)$ & $7(21.2)$ \\
Raid score & $7.9(5.5)$ & $13(28.9)$ & $6(22.2)$ & \\
Proportion, $n(\%)$ & $26(24.8)$ & & & \\
\hline
\end{tabular}

* The RAID score was missing for 1 participant.

Table 3. The occurrence of anxiety (RAID sum score $\geq 11$ ) across time within the groups stratified by level of cognition (MMSE)

\begin{tabular}{llll}
\hline Time point & Sonas & Reading & Control \\
\hline MMSE score 11-20 & & & \\
T0 (at baseline) & $8 / 19(42.1)$ & $4 / 17(23.5)$ & $5 / 21(23.8)$ \\
T1 (at 12 weeks) & $5 / 17(29.4)^{*}$ & $4 / 17(23.5)$ & $6 / 19(31.6)$ \\
T2 (at 24 weeks) & $5 / 17(29.4)^{* *}$ & $3 / 14(21.4)$ & $5 / 18(27.8)$ \\
MMSE score 0-10 & & & \\
T0 (at baseline) & $10 / 29(34.5)$ & $3 / 14(21.4)$ & $6 / 19(31.6)$ \\
T1 (at 12 weeks) & $8 / 29(27.6)$ & $3 / 13(23.1)$ & $4 / 18(22.2)$ \\
T2 (at 24 weeks) & $8 / 28(28.6)$ & $3 / 13(23.1)$ & $2 / 15(13.3)$ \\
\hline
\end{tabular}

Values express $n / N(\%){ }^{*} p=0.428$ (T0 vs. T1), ${ }^{* *} p=0.428$ (T0 vs. T2). There were too few cases to calculate $p$ values.

To our knowledge, this is the largest RCT, with the longest follow-up study, to have investigated the effects of the Sonas program on anxiety and depression.

\section{Anxiety}

The results of this study did not indicate any significant reduction in anxiety in any of the groups, and we found no overall differences between the groups regarding the trend. One reason for the lack of effect could be that the study was underpowered, as the average baseline RAID sum score of the participants was $<10$. This might have limited potentials for a further reduction in anxiety, at both the symptom and disease

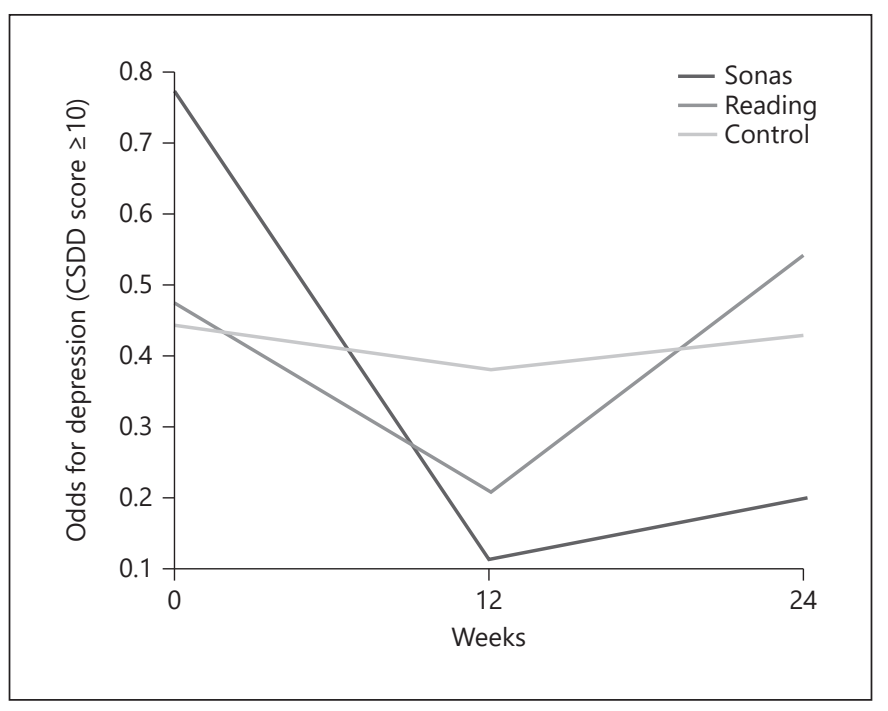

Fig. 3. The course of depression in the 3 groups (GLIMMIX model).

level. However, a nonsignificant reduction in anxiety from baseline (T0) to the 12-week follow-up (T1) was observed in the Sonas group, a finding that should encourage a larger-scale study, i.e., including more PWD with more severe anxiety. Stratifying the groups according to level of cognition (MMSE score), the results did not indicate any effect of cognition on anxiety occurrence in any of the groups at any time point. Again, the main reason was likely due to loss of power. How- 
Table 4. The mean CSDD sum score (SD) and proportion of participants with depression $(C S D D \geq 10)$ in the 3 groups at baseline and at follow-up

\begin{tabular}{lllll}
\hline Time point & Total & Sonas & Reading & Control \\
\hline T0 (at baseline) & & & & \\
Total, $N$ & 120 & 48 & 32 & 40 \\
CSDD score & $8.4(5.7)$ & $8.8(5.0)$ & $7.8(6.0)$ & $8.4(6.2)$ \\
Proportion, $n$ & $45(37.5 \%)$ & $21(43.8 \%)$ & $11(34.4 \%)$ & $13(32.5 \%)$ \\
T1 (at 12 weeks) & & & & \\
Total, $N$ & 113 & 46 & 30 & 37 \\
CSDD score & $6.6(5.1)$ & $6.3(4.2)$ & $5.9(4.7)$ & $7.6(6.2)$ \\
Proportion, $n$ & $23(20.4 \%)$ & $6(13.0 \%)$ & $6(20.0 \%)$ & $11(29.7 \%)$ \\
T2 (at 24 weeks) & & & & \\
Total, $N$ & 105 & 45 & 27 & 33 \\
CSDD score & $6.8(5.3)$ & $6.3(3.9)$ & $7.7(6.2)$ & $6.6(6.0)$ \\
Proportion, $n$ & $29(27.6 \%)$ & $9(20.0 \%)$ & $10(37.0 \%)$ & $10(30.3 \%)$ \\
\hline
\end{tabular}

ever, we find this analysis worthwhile, as the study by Strøm et al. [33] reported that participants with severe dementia in the Sonas group experienced significant improvements in their communication abilities.

\section{Depression}

Even though we found no overall differences in the trend of depressive symptoms between the groups, the participants in the Sonas group experienced a significant reduction in depression. In the reading group, there was a nonsignificant increase in the number of participants with depression at the 24-week follow-up. This may indicate that the extra attention the reading group received was purposeful. It could be that the Sonas program contributed to the reduction in depression. Stratifying the groups according to their cognition level, no significant changes in depression were observed in any group at any time point. Here again, the reason is likely that too few participants with depression were included in each group, subdivided on the level of MMSE scores. As for the mean RAID sum score, the mean CSDD sum score was also low at baseline, indicating that participants had few anxiety and depression symptoms.

The study by Hutson et al. [29] reported some improvement in anxiety and depressive symptoms in both a Sonas and a control group after 7 weeks of intervention. The control group that received treatment as usual showed greater improvement in their anxiety and depressive symptoms than the Sonas group. In contrast, the Sonas group had a greater improvement in symptoms of behavioral disturbances than the control group after 7 weeks. None of the improvements were statisti-
Table 5. The occurrence of depression (CSSD sum score $\geq 10$ ) over time in the 3 groups stratified by level of cognition (MMSE)

\begin{tabular}{llll}
\hline Time point & Sonas & Reading & Control \\
\hline MMSE score 11-20 & & & \\
T0 (at baseline) & $8 / 19(42.1)$ & $3 / 17(17.6)$ & $6 / 21(28.6)$ \\
T1 (at 12 weeks) & $1 / 17(5.9)$ & $2 / 17(11.8)$ & $5 / 19(26.3)$ \\
T2 (at 24 weeks) & $4 / 17(23.5)$ & $5 / 14(35.7)$ & $5 / 18(27.8)$ \\
MMSE score 0-10 & & & \\
T0 (at baseline) & $13 / 29(44.8)$ & $8 / 15(53.3)$ & $7 / 19(36.8)$ \\
T1 (at 12 weeks) & $5 / 29(17.2)$ & $4 / 13(30.8)$ & $6 / 18(73.3)$ \\
T2 (at 24 weeks) & $5 / 28(17.9)$ & $5 / 13(38.5)$ & $5 / 15(33.3)$ \\
\hline
\end{tabular}

Values express $n / N(\%)$. There were too few cases to calculate $p$ values at any point. cally significant, but the study may have been underpowered.

A study by Jackson et al. [31] found an increase in depressive symptoms in the Sonas group after participating in the Sonas session for a period of 8 weeks. The authors could not identify the reason for this but indicated there were qualitative social and psychological benefits for the participants. From the perspective of $\mathrm{NH}$ staff, the Sonas program has some positive effects on mood and interactive activities in $\mathrm{NH}$ residents with dementia [30].

\section{Conclusion}

Although we observed a within-group effect of the Sonas program on depression in $\mathrm{NH}$ residents with moderate to severe dementia, the results of the our study and previous studies are inconclusive with regard to the effects on anxiety and depression in $\mathrm{NH}$ residents with moderate to severe dementia. Participating in the Sonas program may have some positive effects on individual levels which may not be easy to measure with rating scales. We believe that there is a need for larger studies including PWD with severe anxiety and depression, to observe the effects of the Sonas program on anxiety and depression.

\section{Statement of Ethics}

Participants' next-of-kin gave their written informed consent since the participants had moderate to severe dementia and were unable to sign. The study was approved by the Regional Ethics 
Committee of Norway (REK) under the registration No. IRB 0000 1870 and was conducted in accordance with the World Medical Association Declaration of Helsinki [39]. No ethics approval was needed in Ireland, except from the boards of management in each $\mathrm{NH}$, and they approved [33].

\section{Conflict of Interest Statement}

The authors declare there were no conflicts of interest.

\section{Funding Sources}

There was no funding.

\section{Author Contributions}

B.S.S. collected the data. K.E., A.R.G., and B.S.S. contributed to the design of the study. J.Š.B. conducted the statistical analysis and guided the interpretation of the data. A.R.G. wrote the paper. All listed authors supervised the writing of the paper and approved the final version for publication.

\section{References}

1 Barca ML, Engedal K, Laks J, Selbaek G. A 12 months follow-up study of depression among nursing-home patients in Norway. J Affect Disord. 2010 Jan;120(1-3):141-8.

2 Goyal AR, Bergh S, Engedal K, Kirkevold M, Kirkevold Ø. Anxiety, Anxiety Symptoms, and Their Correlates in Persons with Dementia in Norwegian Nursing Homes: A Cause for Concern. Dement Geriatr Cogn Disord. 2017;43(5-6):294-305.

3 Tori K, Kalligeros M, Nanda A, Shehadeh F, van Aalst R, Chit A, et al. Association between dementia and psychiatric disorders in long-term care residents: an observational clinical study. Medicine (Baltimore). 2020 Jul;99(31):e21412.

4 van der Linde RM, Dening T, Stephan BC, Prina AM, Evans E, Brayne C. Longitudinal course of behavioural and psychological symptoms of dementia: systematic review. $\mathrm{Br}$ J Psychiatry. 2016 Nov;209(5):366-77.

5 Wolitzky-Taylor KB, Castriotta N, Lenze EJ, Stanley MA, Craske MG. Anxiety disorders in older adults: a comprehensive review. Depress Anxiety. 2010 Feb;27(2):190-211.

6 Seignourel PJ, Kunik ME, Snow L, Wilson N, Stanley M. Anxiety in dementia: a critical review. Clin Psychol Rev. 2008 Oct;28(7):107182.

7 Seitz D, Purandare N, Conn D. Prevalence of psychiatric disorders among older adults in long-term care homes: a systematic review. Int Psychogeriatr. 2010 Nov;22(7):1025-39.

8 Calleo JS, Kunik ME, Reid D, Kraus-Schuman C, Paukert A, Regev T, et al. Characteristics of generalized anxiety disorder in patients with dementia. Am J Alzheimers Dis Other Demen. 2011 Sep;26(6):492-7.

9 Goyal AR, Bergh S, Engedal K, Kirkevold M, Kirkevold Ø. The course of anxiety in persons with dementia in Norwegian nursing homes: A 12-month follow-up study. J Affect Disord. 2018 Aug;235:117-23.
10 Porter VR, Buxton WG, Fairbanks LA, Strickland T, O'Connor SM, Rosenberg-Thompson $S$, et al. Frequency and characteristics of anxiety among patients with Alzheimer's disease and related dementias. J Neuropsychiatry Clin Neurosci. 2003;15(2):180-6.

11 Majić T, Pluta JP, Mell T, Treusch Y, Gutzmann H, Rapp MA. Correlates of agitation and depression in nursing home residents with dementia. Int Psychogeriatr. 2012 Nov;24(11):1779-89.

12 American Psychiatric Association. Diagnostic and statistical manual of mental disorders: DSM-5. Washington, DC: American Psychiatric Association; 2013. p. 947.

13 Nordhus IH. Manifestations of Depression and Anxiety in Older Adults. In: Woods R, Clare L, editors. Handbook of the Clinical Psychology of Ageing. 2nd ed. John Wiley \& Sons, Ltd; 2008. pp. 95-110.

14 WHO. ICD-11 : International Classification of Diseases 11th Revision. WHO; 2018.

15 Cooper C, Balamurali TB, Livingston G. A systematic review of the prevalence and covariates of anxiety in caregivers of people with dementia. Int Psychogeriatr. 2007 Apr;19(2):175-95.

16 Gibbons LE, Teri L, Logsdon R, McCurry SM, Kukull W, Bowen J, et al. Anxiety symptoms as predictors of nursing home placement in patients with Alzheimer's disease. J Clin Geropsychol. 2002 Oct;8(4):335-42.

17 Barca ML, Selbaek G, Laks J, Engedal K. Factors associated with depression in Norwegian nursing homes. Int J Geriatr Psychiatry. 2009 Apr;24(4):417-25.

18 Spector A, Orrell M. Using a biopsychosocial model of dementia as a tool to guide clinical practice. Int Psychogeriatr. 2010 Sep;22(6): 957-65.

19 Holmes SE, Esterlis I, Mazure CM, Lim YY, Ames D, Rainey-Smith S, et al.; Australian Imaging, Biomarkers and Lifestyle Research Group. Trajectories of depressive and anxiety symptoms in older adults: a 6-year prospective cohort study. Int J Geriatr Psychiatry. 2018 Feb;33(2):405-13.
20 Kitwood T. The experience of dementia. Aging Ment Health. 1997 Feb;1(1):13-22.

21 Zwijsen SA, van der Ploeg E, Hertogh CM. Understanding the world of dementia. How do people with dementia experience the world? Int Psychogeriatr. 2016Jul;28(7):106777.

22 Alzheimer's Association. 2015 Alzheimer's disease facts and figures. Alzheimers Dement. 2015 Mar;11(3):332-84.

23 World Health Organization. Global action plan on the public health response to dementia2017-2025; 2017.

24 Seitz DP, Gill SS, Herrmann N, Brisbin S, Rapoport MJ, Rines J, et al. Pharmacological treatments for neuropsychiatric symptoms of dementia in long-term care: a systematic review. Int Psychogeriatr. 2013 Feb;25(2):185203.

25 Wang J, Yu JT, Wang HF, Meng XF, Wang C, Tan CC, et al. Pharmacological treatment of neuropsychiatric symptoms in Alzheimer's disease: a systematic review and meta-analysis. J Neurol Neurosurg Psychiatry. 2015 Jan;86(1):101-9.

26 Cohen-Mansfield J. Non-pharmacological interventions for persons with dementia: what are they and how should they be studied? Int Psychogeriatr. 2018 Mar;30(3):281-3.

27 Orgeta V, Qazi A, Spector A, Orrell M. Psychological treatments for depression and anxiety in dementia and mild cognitive impairment: systematic review and meta-analysis. Br J Psychiatry. 2015 Oct;207(4):293-8.

28 Strøm BS. Overview of research on the Sonas program. Dublin: Engaging Dementia; 2020.

29 Hutson C, Orrell M, Dugmore O, Spector A. Sonas: a pilot study investigating the effectiveness of an intervention for people with moderate to severe dementia. Am J Alzheimers Dis Other Demen. 2014 Dec;29(8):696-703.

30 Dugmore O. The impact and process of Sonas apc for dementia: views from care home staff. D Clin Psych. Thesis (volume1). London: University College London; 2012.
Anxiety and Depression and the Sonas Program
Dement Geriatr Cogn Disord Extra 2021;11:151-158 DOI: $10.1159 / 000516804$ 
31 Jackson GA, Sterling R, Russell K, Templeton G. A multisensory programme: evaluating effects on agitation. Nurs Resident Care. 2003 Mar;5(3):126-9.

32 Polit DF, Beck CT. Nursing research: generating and assessing evidence for nursing practice. Philadelphia. Wolters Kluwer Health. 2012;XIV:802.

33 Strøm BS, Engedal K, Benth JS, Grov EK. Effect of the sonas programme on communication in people with dementia: a randomized controlled trial. Dement Geriatr Cogn Disord Extra. 2017 Apr;7(1):122-35.
34 Folstein MF, Folstein SE, McHugh PR. "Minimental state". A practical method for grading the cognitive state of patients for the clinician. J Psychiatr Res. 1975 Nov;12(3):189-98.

35 Tombaugh TN, McIntyre NJ. The mini-mental state examination: a comprehensive review. J Am Geriatr Soc. 1992 Sep;40(9):92235.

36 Shankar K, Walker M, Frost D, Orrell M. The development of a valid and reliable scale for rating anxiety in dementia (RAID). Aging Ment Health. 1999;3(1):39-49.
37 Alexopoulos GS, Abrams RC, Young RC, Shamoian CA. Cornell Scale for Depression in Dementia. Biol Psychiatry. 1988 Feb; 23(3):271-84.

38 Barca ML, Engedal K, Selbaek G. A reliability and validity study of the cornell scale among elderly inpatients, using various clinical criteria. Dement Geriatr Cogn Disord. 2010;29 (5):438-47.

39 World Medical Association. World Medical Association Declaration of Helsinki: ethical principles for medical research involving human subjects. JAMA. 2013 Nov;310(20): $2191-4$ 